\title{
Photoinduced supramolecular chirality and spontaneous surface patterning in high-performance azo materials
}

\author{
Sekvan Bagatur and Thomas Fuhrmann-Lieker ${ }^{*}$
}

\begin{abstract}
In this work, two photo-structuring processes, photo induced spontaneous surface structuring (PSSP) and photo induced supramolecular chirality (PSC) are applied to high-performance azo polymers. We demonstrate higher induced optical rotation than described previously in other reports and show that self-organized surface morphologies are not detrimental for the formation of PSC.
\end{abstract}

Keywords: Azomaterials, Supramolecular chirality, Spontaneous patterning

\section{Introduction}

The application of light for lithography, i.e. structuring processes of solid materials, is an established method in research and development of two and three-dimensional structuring. Materials used for these purposes are called "photoadressable" due to their reversible or irreversible change in physical and/or chemical behavior, such as viscosity, solubility, mechanical parameters (e.g. shear modulus, Young's modulus, etc.) and optical constants (birefringence, circular dichroism, etc.) [1-10]. Azo dyes constitute a well investigated material class that represent good candidates for optoelectronic devices based on organic molecules [1, 2]. Surface corrugation [3-5], holographic data storage [6-10], grating couplers [11], and as output control layers in organic light-emitting diodes (OLED) [12] are only few examples. Even the application in biological systems for control of biochemical processes and manipulation of physical properties is under consideration $[13,14]$.

The characteristic of azo compounds is its functional azo $-\mathrm{N}=\mathrm{N}$ - double bond with organic groups on each nitrogen atom. These substitutes stand either in trans or in cis-configuration. Upon irradiation at an appropriate wavelength in the range of absorption, the thermodynamically stable trans-configuration isomerizes

*Correspondence: th.fuhrmann@uni-kassel.de

Macromolecular Chemistry and Molecular Materials, Institute of Chemistry, Universität Kassel, Kassel, Germany to the cis-type. The reverse isomerization can be performed either photochemically or thermally (Fig. 1). Interestingly, photoisomerization depends on the orientation between the polarization state $P$ of an incident light beam and the transition dipole moment $M$ of an azo group, which is roughly parallel to the $-\mathrm{N}=\mathrm{N}$ - double bond (photoselection). The probability of isomerization decreases from $0^{\circ}\left(P \| M\right.$, as seen in Fig. 1a) to $90^{\circ}(P \perp M$, as seen in Fig. 1b). The repeated change of configuration during the continuing irradiation causes a reorientation - called photoorientation - of azo molecules until $M$ is perpendicular to $P$. During this photoorientation process, the mobility of molecules at the spot of irradiation is increased and viscosity decreased, even though the temperature is well below $T_{\mathrm{g}}$. Consequently, driving by internal stresses, an active light induced mass transport can occur and leads to macroscopic change of the surface or bulk of an azo layer [5]. Probably the first work on photomechanical effects of azo materials was from Blair et al. [15], whereas the first photoinduced periodic surface structures, called surface relief gratings (SRG), on azo layers were reported by Rochon et al. [3] and Kim et al. [4] independently. While SRG's are induced by two interfering Gaussian beams at an appropriate wavelength and a certain polarization state, the usage of a single polarized beam induces bump structures on the surface, called photoinduced spontaneous surface patterning (PSSP), as reported by Hubert et al. [16-18]. If irradiation occurs with linearly polarized light (LPL), hexagonally ordered bump patterns arise and, if the 

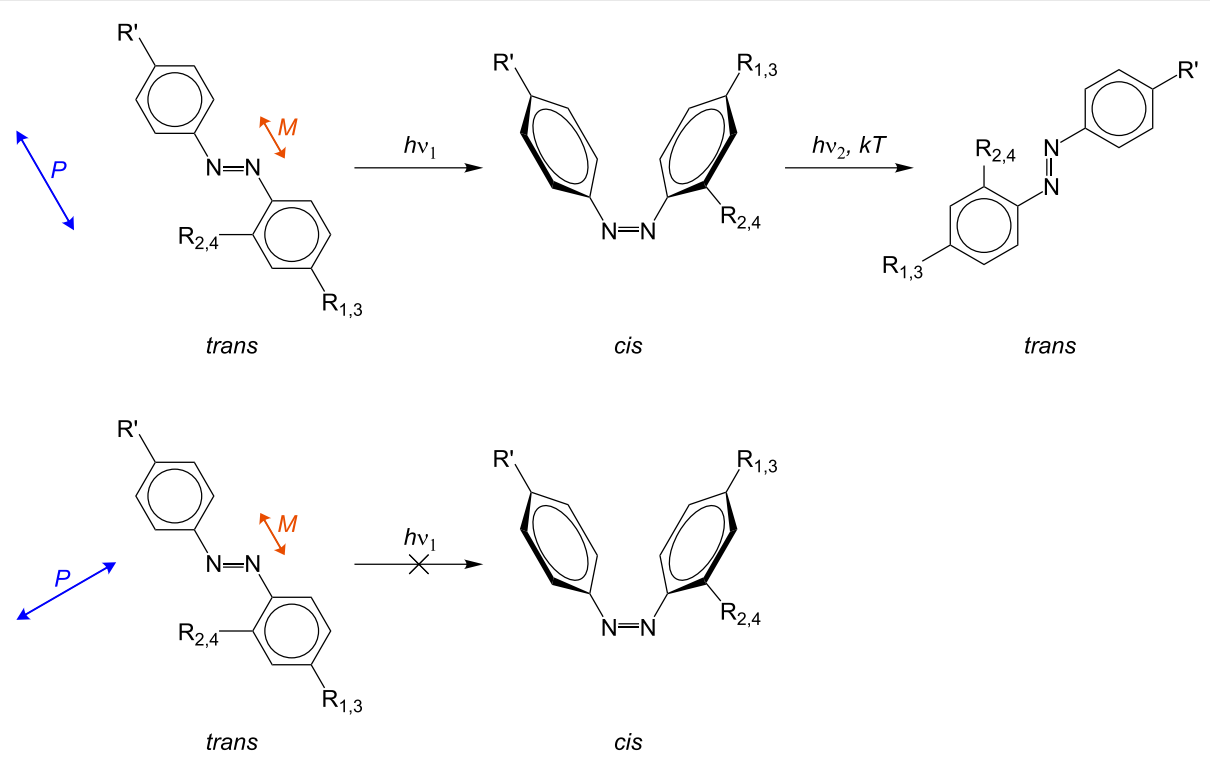

Fig. 1 Chemical equation of photo-isomerization of an azo molecule. The light induced isomerization of an azo molecule depends on the orientation between transition dipole moment $M$ and polarization state $P$ of incident light. The probability of isomerization decreases from $0^{\circ}$ $(P \| M)$ to $90^{\circ}(P \perp M)$

incident light beam is circularly polarized (CPL), random patterns are formed.

In addition to surface structuring, elliptically polarized light (EPL) affects the azo material within the bulk itself, namely, a helical chiral arrangement of azo groups can be light-induced along the film normal with either right or left handedness depending on the chirality of incident light. The structure can be regarded as a helix of locally linearly birefringent planes that drills itself into the material with supramolecular pitch. This so-called photoinduced supramolecular chirality (PSC) of achiral polymers was reported by Kim et al. for the first time [19, 20]. They inscribed helices with azimuthal rotation $\Delta \psi$ of $4^{\circ}$ and $4.5^{\circ}$, respectively. The group of Mazaheri reported supramolecular chirality in another azo dye with a higher value of $\Delta \psi=10^{\circ}$ [21].

Here, we apply PSSP and PSC to a special class of high-performance polymers that are known for their high optical birefringence [6]. With these materials, we aim at improving the amount of optical rotation that can be induced in a thin film. We investigate whether surface patterns evolve simultaneously and they have an impact on the amount of optical rotation.

The materials are chosen at representatives of three different classes: Polymer $\mathbf{1}$ is an amorphous two-core sidechain polymer, Polymer $\mathbf{2}$ is a liquid-crystalline three-core polymer, and Polymer $\mathbf{3}$ is a mixed two-core and threecore liquid-crystalline polymer. The molecular structural formulas of the used materials and their absorption spectra are shown in Figs. 2 and 3, respectively. The functional groups for each polymer, listed in Table 1, represent strong push-pull systems. Polymers 2 and $\mathbf{3}$ are characterized by a side chain with three connected aromatic cores and therefore high anisotropic polarizability.

We investigate the response of thin layers on glass substrates to a single, coherent and monochromatic Gaussian beam at an defined excitation wavelength $\left(\lambda_{\text {exc. }}=473 \mathrm{~nm}\right)$ with different polarization states, namely LPL, CPL, and EPL. According to [19] and [20], PSC occurs only by using EPL. Therefore, we expect only PSSP for linear and circular polarization, and both PSSP and PSC with elliptical polarization.

The setup for excitation is shown in Fig. 4a. For analyzing the optical rotation of PSC, a self-made polarimeter using a HeNe-laser $(\lambda=633 \mathrm{~nm})$ with EPL was used as seen in Fig. $4 \mathrm{~b}$. The ellipticity and azimuthal angle of excitation and analysis beam were adjusted to be the same. In Fig. 5, the corresponding angles are defined: The incident EPL-beam can be described by a minor $a$ and major axis $b$ with the ellipticity $\epsilon=\arctan a / b$. Here, $b$ is in the $x-y$-plane if we assume the direction of light propagation represents the $z$-axis in a coordinate system. If we define the angle between $x$-axis and $b$ as the azimuthal angle $\psi$, we are able to determine the azimuthal rotation $\Delta \psi$ of $b$ in $x-y$-plane after EPL propagation through the optically active sample. This is possible with a rotatable polarizer placed after the sample. In our setup, the rotation was done with steps of $1^{\circ}$. Therefore, the uncertainty of azimuthal rotation $\Delta \psi$ amounts to $\delta \psi=0.5^{\circ}$. 
<smiles>CNc1ccc(NC(=O)c2ccc(OCCOC(=O)C(C)(C)CC(C)(C)C)cc2)cc1</smiles><smiles>Nc1ccc(Br)cc1</smiles>

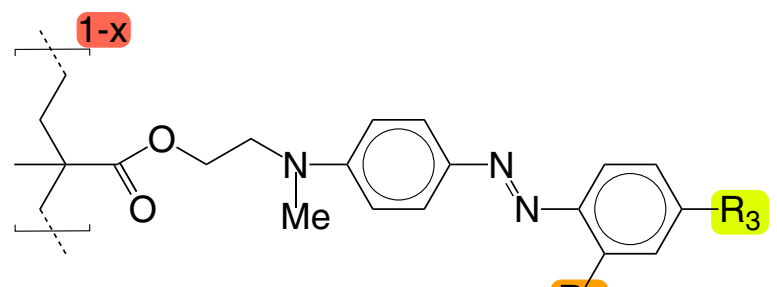

$\mathrm{R}_{4}$

Fig. 2 Basic representation of azo polymers used in this work. $x$ denotes the relative percentage of each side chain. The polymers were synthesized at Bayer AG by Dr. Kostromine in the 1990's

In order to characterize the time dependent development, we varied the irradiation times, 30 and $60 \mathrm{~min}$ for PSSP, and from 1 to 70 min for PSC, respectively.

\section{Results and discussion}

The absorption spectra in Fig. 3 show one maximum for polymer 1 in the range between $\sim 355-600 \mathrm{~nm}$ and one maximum for polymer 2 in the range of $\sim 320-550 \mathrm{~nm}$. Polymer 3 (blue curve) has two maxima due to the three and two-core side-chain in the copolymer. We assign by comparison with other polymers of this series [6] the maximum at $\sim 375 \mathrm{~nm}$ to the three-core side-chain and the maximum at $\sim 512 \mathrm{~nm}$ to the two-core side-chain. As seen in the absorption spectra, the excitation wavelength $\lambda_{\text {exc. }}$ is at the absorption edge in case of 2 and approximately at the two-core absorption maxima of $\mathbf{1}$ and $\mathbf{3}$. The analysis wavelength of $633 \mathrm{~nm}$ is not in the absorption range in all cases and therefore does not affect any azo material optically.

After preparation, all spin-coated samples showed inplane isotropic behavior under the polarization microscope.

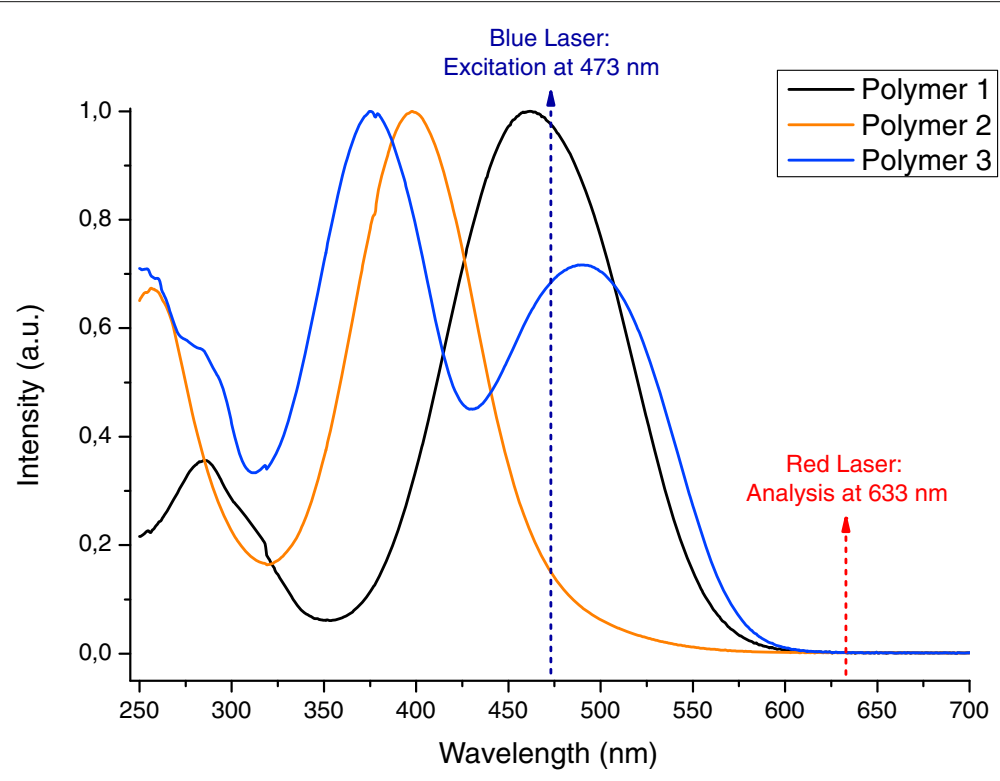

Fig. 3 Absorption spectra of used azo materials (solved in THF) and wavelengths of used light sources 
Table 1 List of functional groups of used azo polymers in this work and their percentage for each side chain

\begin{tabular}{|c|c|c|c|c|c|c|c|}
\hline \multirow[t]{2}{*}{ Polymer } & \multirow[t]{2}{*}{$T_{\mathrm{g}}\left[{ }^{\circ} \mathrm{C}\right]$} & \multicolumn{2}{|c|}{ Three cores } & \multicolumn{2}{|c|}{ Two cores } & \multirow[t]{2}{*}{$x[\%]$} & \multirow[t]{2}{*}{ Comments } \\
\hline & & $\mathrm{R}_{1}$ & $\mathrm{R}_{2}$ & $\mathrm{R}_{3}$ & $\mathrm{R}_{4}$ & & \\
\hline 1 & - & - & - & $\mathrm{NO}_{2}$ & $\mathrm{H}$ & 0 & Homopolymer \\
\hline 2 & 147 & $\mathrm{CN}$ & $\mathrm{CN}$ & - & - & 100 & Homopolymer \\
\hline 3 & 116 & $\mathrm{CN}$ & $\mathrm{H}$ & $\mathrm{CN}$ & $\mathrm{CN}$ & 60 & Copolymer \\
\hline
\end{tabular}

In the following, we first discuss PSSP with suppression of PSC by using LPL and CPL. Then, we discuss PSC with suppression of PSSP by irradiation from the substrate side. Finally, we treat the general case with both structuring mechanisms present, i.e. using EPL and irradiation from the polymer side.

\section{Photoinduced spontaneous surface patterning}

Irradiation of the given azo polymers with linear or circular polarization induces bump patterns on the surfaces, similar to those observed in [17]. For example, the sample 1 with the layer thickness of $d=101 \mathrm{~nm}$ shows well pronounced hexagonal ordered bump patterns (Fig. 6a, b) upon irradiation with LPL for 30 (Fig. 6a) and $60 \mathrm{~min}$ (Fig. 6b). The Fast-Fourier-Transform (FFT) image clearly confirms the hexagonally structured pattern on the surface. The mean distance between each bump for both irradiation durations is similar and amounts to $\Lambda=420 \mathrm{~nm}$ for $30 \mathrm{~min}$ irradiation and $\Lambda=406 \mathrm{~nm}$ for $60 \mathrm{~min}$ irradiation (see Table 2). The modulation depths $h$, i.e. depth of the bumps, does not change significantly with longer exposure times. Irradiation with CPL (Fig. 6c, d), on the other hand, leads to randomly distributed bump patterns with similar distances of $\Lambda=421 \mathrm{~nm}(30 \mathrm{~min})$ and $\Lambda=421 \mathrm{~nm}(60 \mathrm{~min})$. Again, the modulation depth does not change significantly for an exposure time of 30 (Fig. 6c) and $60 \mathrm{~min}$ (Fig. 6d). Additionally, we observed a photo-bleaching effect for our samples, as described by Hubert et al. [18] and Mazaheri et al. [22], too. Even though the change of transmittance for photo-bleached areas was not measured by us, macroscopically an increment was observed due to decolorization and higher transparency of these areas.

Since thicker films are required later for PSC measurements, we now discuss the influence of the film thickness on the pattern morphology. In case of 1 with $d=493 \mathrm{~nm}$, the bump pattern disappears and rib structures were produced instead. It seems that smaller bumps are fused to larger parallel ribs, which is more obvious at longer irradiation times (Fig. 6f). Irradiation of the same sample with circular polarization (Fig. 6g, h) leads to morphologies
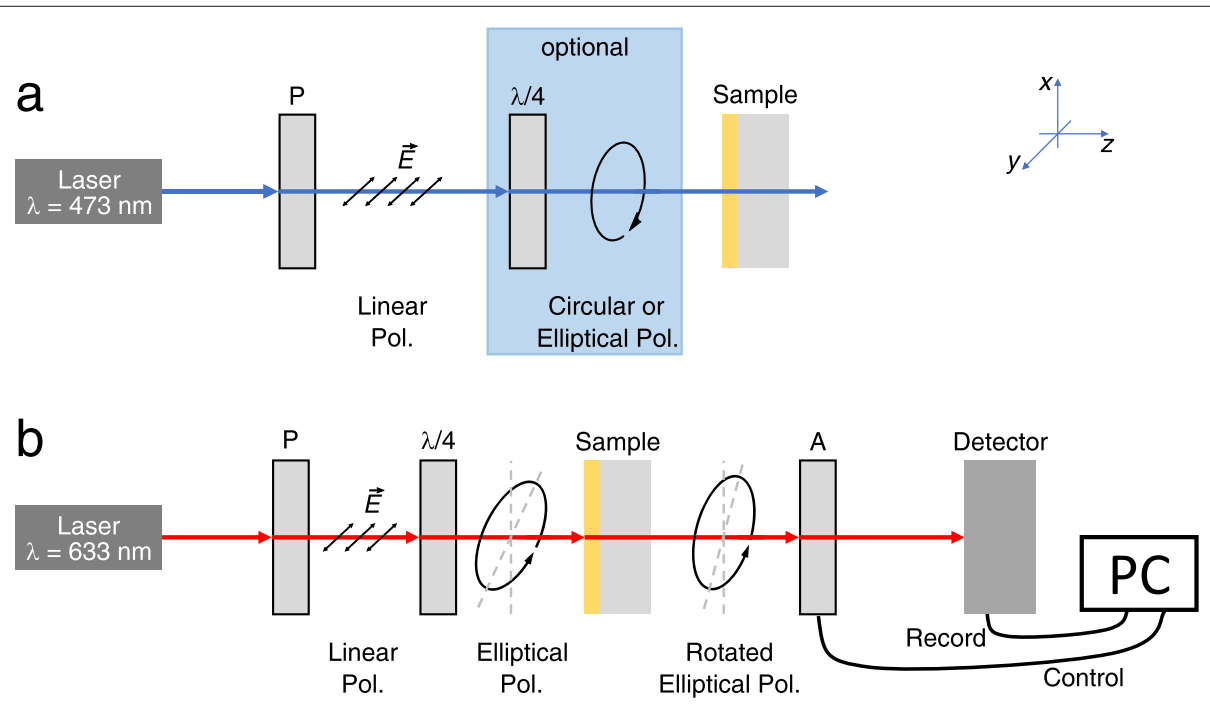

Fig. 4 Schematic representation of the setup for a) PSSP and PSC, respectively, as well as for b) analysis of PSC. a The azo sample was treated by a DPSS-laser at $\lambda_{\text {exc. }}=473 \mathrm{~nm}$ with either linear or circular polarization for PSSC and with elliptical polarization for PSC. Linear polarization was assured by a polarizer (P), circular and elliptical polarization by a quarter-waveplate. $\mathbf{b}$ The analysis of the supramolecular chiral structures was done by a self-made polarimeter consisting of a HeNe-laser at $\lambda=633 \mathrm{~nm}$, a polarizer $\mathrm{P}$, quarter-waveplate, a rotatable analyzer $\mathrm{A}$ (around $z$-axis) and a diode detector, which detects the intensity EPL dependent on the position of A. The rotation of A was controlled by a computer with a self-written LabVIEW program, which also collects intensity data from detector 


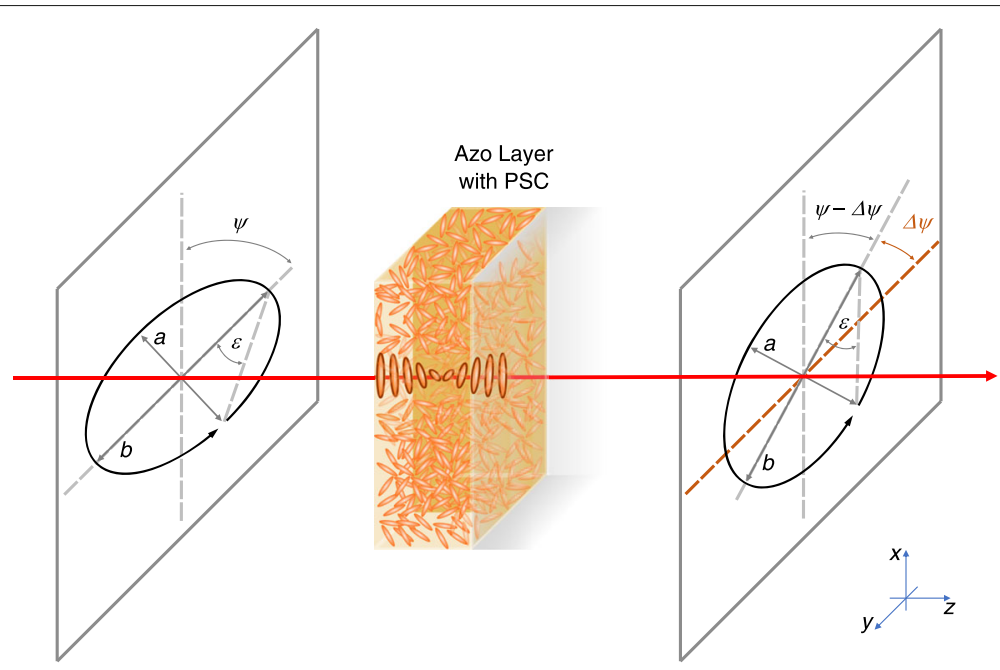

Fig. 5 Orientation of polarization states before and after transmission. The elliptically polarized beam propagates along $z$-axis, while minor $a$ and major axis $b$ of the electric field are in $x-y$-plane. After a beam at a non-absorbing wavelength $(\lambda=633 \mathrm{~nm})$ is transmitted through a sample with photo-induced supramolecular chirality, $b$ rotates by an angle of $\Delta \psi \cdot \epsilon$ denotes the ellipticity

resembling the patterns of the thinner samples. For both exposure times of 30 (Fig. 6g) and $60 \mathrm{~min}$ (Fig. 6h) randomly distributed bump patterns were obtained. However, the sphere-like bumps are more heterogenous in size. The longer the exposure time the higher is the disorder and also the modulation depth $h$.

Results for the other two polymers ( 2 and $\mathbf{3}$ ) are shown exemplarily in Fig. 7a and b for the case of thick films, LPL and 60 min irradiation. Compared with Fig. 6f, homopolymer 2 with a thickness of $d=580 \mathrm{~nm}$ does not show well pronounced patterns and exhibits the lowest $h$ among all used samples (Fig. 7a). However, polymer 3 under the same irradiation conditions shows a well preserved ordered bump pattern, even at thicknesses as high as $d=886 \mathrm{~nm}$ (Fig. 7b). The FFT image confirms a periodic and approximately hexagonal pattern with deep modulation depth.

Average distances $\Lambda$ and estimated modulation depths $h$ of all polymer samples given in Fig. 6 are summarized in Table 2.
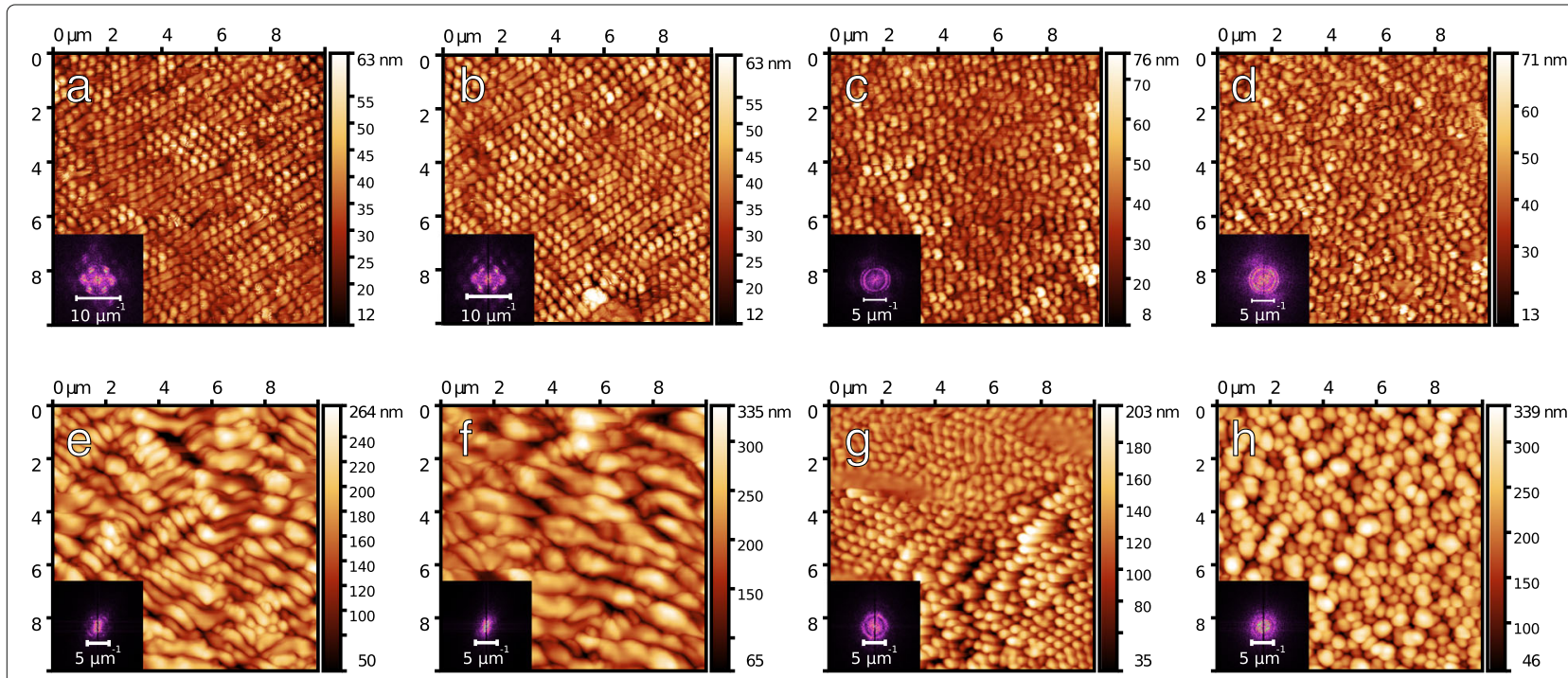

Fig. 6 AFM and FFT images of sample $\mathbf{1}$ with $d=101 \mathrm{~nm}(\mathbf{a}-\mathbf{d})$ and $d=493 \mathrm{~nm}(\mathbf{e}-\mathbf{h})$. The samples $\mathbf{a}), \mathbf{c})$, e), and $\mathbf{g}$ ) have been irradiated for $30 \mathrm{~min}$ and $\mathbf{b}), \mathbf{d}), \mathbf{f})$, and $\mathbf{h})$ for 60 min with linear polarization $(\mathbf{a}, \mathbf{b}, \mathbf{e}, \mathbf{f})$ and circular polarization $(\mathbf{c}, \mathbf{d}, \mathbf{g}, \mathbf{h})$. The bump structures are very well pronounced and confirmed by their FFT images (a-d). In case of irradiation with CPL the bump structures are not hexagonal ordered as in $\mathbf{a}$ ) and $\mathbf{b}$ ), but randomly distributed with an average distance $\Lambda$ between the bumps (see Table 2). In $\mathbf{e}$ ) and $\mathbf{f}$ ), the bump structures seem like fused to parallel rib structures and have similar distances 
Table 2 Summarized results of average distances of periodic patterns $\Lambda$ and estimated modulation depths $h$

\begin{tabular}{|c|c|c|c|c|c|c|c|c|c|}
\hline \multirow[t]{3}{*}{ Polymer } & \multirow[t]{3}{*}{ Thickness $d[\mathrm{~nm}]$} & \multicolumn{4}{|c|}{ Linear polarization } & \multicolumn{4}{|c|}{ Circular polarization } \\
\hline & & \multicolumn{2}{|c|}{$30 \mathrm{~min}$} & \multicolumn{2}{|c|}{$60 \mathrm{~min}$} & \multicolumn{2}{|c|}{$30 \mathrm{~min}$} & \multicolumn{2}{|c|}{$60 \mathrm{~min}$} \\
\hline & & $\Lambda[\mathrm{nm}]$ & $h[\mathrm{~nm}]$ & $\Lambda[\mathrm{nm}]$ & $h[\mathrm{~nm}]$ & $\Lambda[\mathrm{nm}]$ & $h[\mathrm{~nm}]$ & $\Lambda[\mathrm{nm}]$ & $h[\mathrm{~nm}$ \\
\hline \multirow[t]{2}{*}{1} & 101 & $401.9 \pm 2.4$ & 51 & $406.3 \pm 1.1$ & 51 & $412.7 \pm 2.4$ & 68 & $420.6 \pm 1.4$ & 58 \\
\hline & 493 & $434.9 \pm 1.3$ & 214 & $930.5 \pm 9.4$ & 270 & $412.9 \pm 2.3$ & 168 & $1472.5 \pm 31.3$ & 293 \\
\hline 2 & 580 & & & $412.7 \pm 0.9$ & 22 & & & & \\
\hline 3 & 886 & & & $440.6 \pm 3.7$ & 70 & & & & \\
\hline
\end{tabular}

While values for $\Lambda$ were obtained by using AFM images and calculations with a Python program, the values for $h$ were estimated by a heuristic determination of the height profile with tails cut off. The listed values are the difference of maximum and minimum value in the color range map of each AFM image (see Figs. 6 and 7 )

Additionally, in order to investigate the response to strongly focused light with higher intensity, a lens (focal length $f=12.5 \mathrm{~mm}$ ) was placed between polarization filter or retarder, respectively, and sample. Figure 8 shows the results for Polymer 1 with $d=493 \mathrm{~nm}$ in the focal point of LPL (Fig. 8a) and CPL (Fig. 8b). Due to wavefront distortions and interference effects, concentric patterns with high irregularity are observed.

\section{Photoinduced supramolecular chirality}

For the investigation of PSC, we first minimized the influence of PSSP by irradiation of the sample with EPL from the glass side, as Hubert et al. have found out that in this case PSSP does not occur or is very weak, respectively [18].

Depending on exposure time and ellipticity $\epsilon$ (see Fig. 5), a helical arrangement of the azo molecules is induced along the film normal in $z$-direction. Depending on the penetration depth due to absorption, the order is expected to decrease within the bulk for thicker films. However, because of the reorientation process the penetration depth will depend on irradiation time to some degree. We measured the quantity of the supramolecular chirality with the self-made polarimeter by measuring the angle-dependent intensity of transmitted EPL and therefore determining the azimuthal rotation, i.e. the change of azimuthal angle.

First, we confirmed that PSSP is relatively weakly under these conditions as can be seen in Fig. 9. The AFM images do not show pronounced surface structures with high modulation depths. However, in some locations but not throughout the film elongated small and big bubbles occured, probably because of stress release since they all are elongated in the same direction (Fig. 9b and d). Also in the case of relative smooth surfaces, some anisotropy, which can be described as in-plane chirality, can be detected (Fig. 9a and c).

The development of PSC in dependence on exposure time $(1,5,10,30$, and $70 \mathrm{~min})$ and layer thickness (three different $d$ ) was investigated by using samples of all polymers: An ellipticity of $\epsilon=15^{\circ}$ was chosen for all samples. The azimuthal angle of incident EPL amounts to $\psi=14^{\circ}$ measured through a cleaned and uncoated glass slide. However, the light transmitted through an untreated azo-layer shows often a changed azimuthal angle dependent on the material and layer thickness. This was obtained in the reference publications [19] and [20], too. It might be caused by inhomogeneities of the
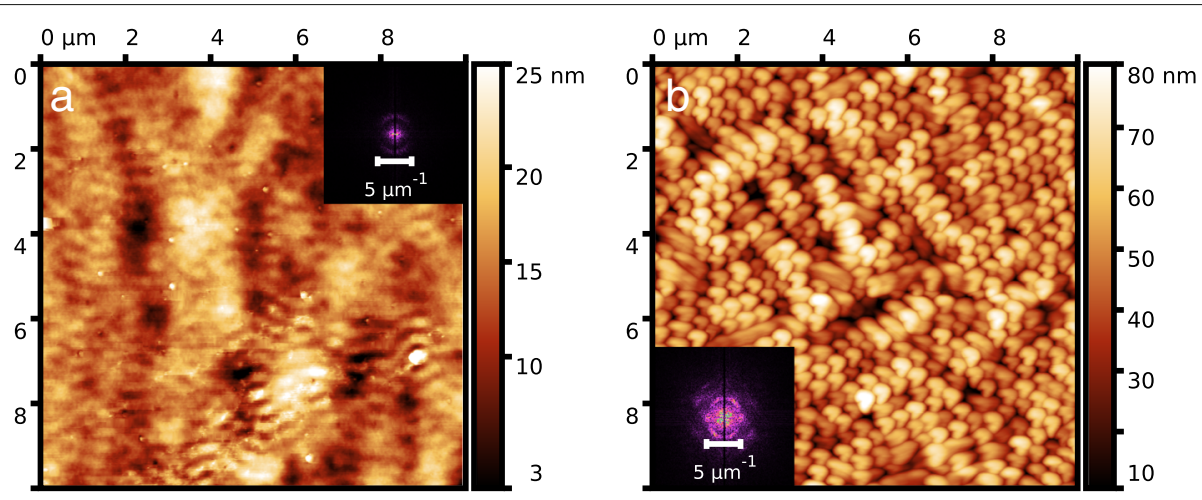

Fig. 7 AFM and FFT images of samples of polymer a) $\mathbf{2}$ and b) 3. a Sample 2: Irradiation with LPL for 60 min. Even though the structuring looks very weak, the FFT-image shows an anisotropy and ordered pattern with $\Lambda=413 \mathrm{~nm}$. b Sample 3: Irradiation for 60 min with linear polarization. The bump structures are very well pronounced and have an arrow-like structure. The bumps seem to be oriented, which is also confirmed by its FFT-image. Additionally, the arrow-like bumps are all oriented in the same direction 

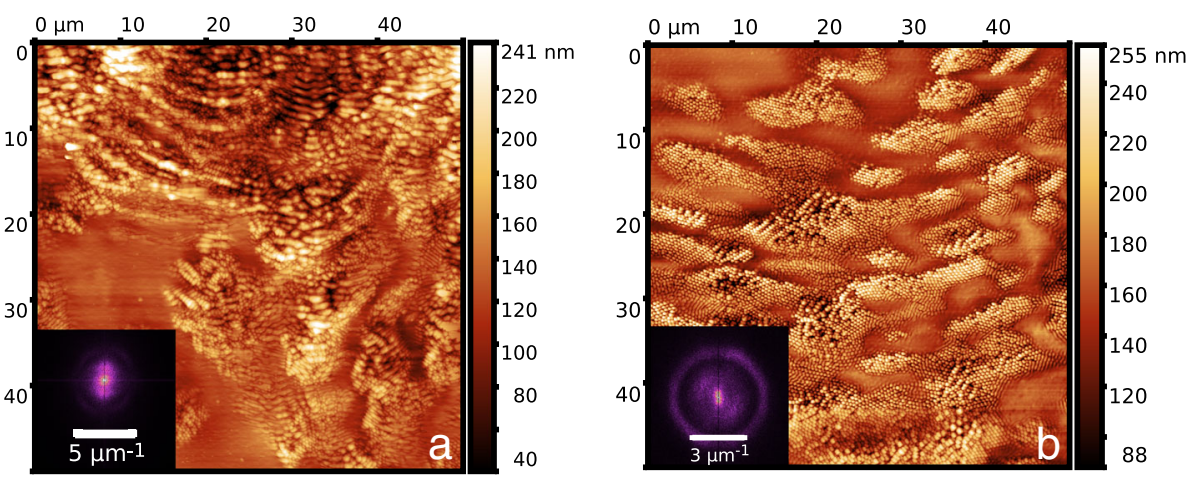

Fig. 8 AFM, FFT and 3D images of sample 1 with $d=493 \mathrm{~nm}$. a By placing a lens with $f=12.5 \mathrm{~mm}$ with the surface of sample in focal point, interference pattern caused by the lens is visible on the surface. This confirms the dependency on light intensity for structuring. This sample was irradiated for 30 min with linear polarization. $\mathbf{b}$ Here, the situation is the same as in a) with the lens, but with CPL

spin-coated azo layers. A large-scale birefringence can be excluded, however, according to polarization microscopy. In our work, azimuthal rotation $\Delta \psi$ is thus always given relative to the transmitted azimuthal angle before irradiation of the sample.

The obtained results, plotted in Fig. 10 and listed in Table 3, show azimuthal rotation with an increase for longer exposure times and layer thicknesses. The highest value for azimuthal rotation of $\Delta \psi=37^{\circ}$ was achieved in case of 2 with a thickness of $d=888 \mathrm{~nm}$. The results demonstrate in some cases higher $\Delta \psi$ than in [20] and [21].

In order to visualize the development of PSC, azimuthal rotation for each sample is plotted against exposure time
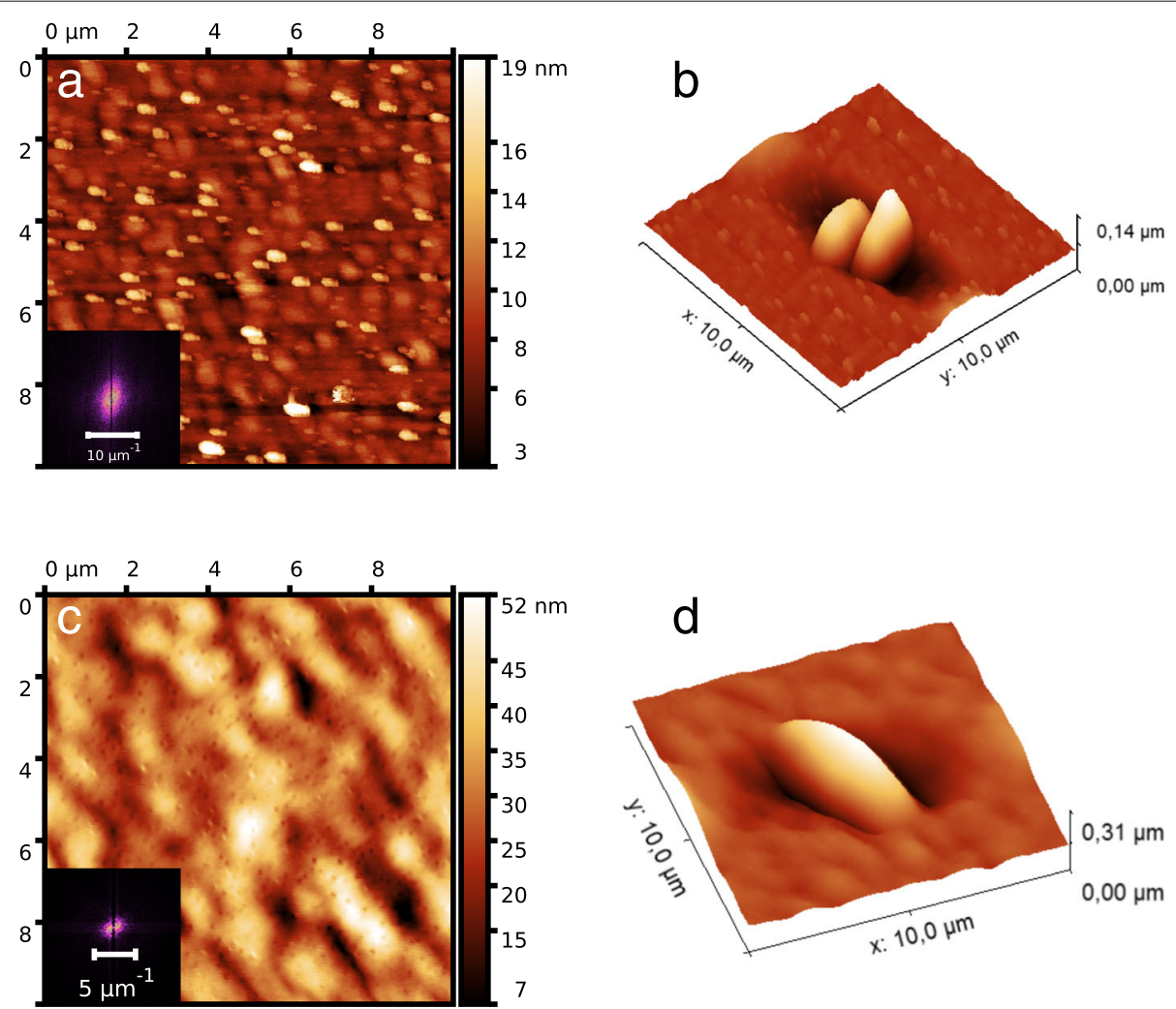

Fig. 9 2D and 3D-AFM images of samples of polymer 3. a, b) Surface of sample with a thickness of $d=330 \mathrm{~nm}$ and $\mathbf{c}$, d) $d=623 \mathrm{~nm}$. Both samples were irradiated with EPL $\left(\epsilon=15^{\circ}\right)$ for 70 min (right). The surfaces are clearly different and PSSP after irradiation is very weakly pronounced. As already mentioned, some small and big bubbles are visible on the surface in $\mathbf{b}$ ) and $\mathbf{d}$ ) 
Table 3 Results for polarimetry measurements in case of irradiating the glass side by EPL with an ellipticity of $\epsilon=15^{\circ}$

\begin{tabular}{|c|c|c|c|c|c|c|c|}
\hline \multirow[t]{2}{*}{ Polymer } & \multirow[t]{2}{*}{$d[\mathrm{~nm}]$} & \multirow{2}{*}{$\begin{array}{l}0 \text { min } \\
\psi\left[^{\circ}\right]\end{array}$} & \multirow{2}{*}{$\frac{1 \min }{\Delta \psi\left[^{\circ}\right]}$} & \multirow{2}{*}{$\frac{5 \min }{\Delta \psi\left[^{\circ}\right]}$} & \multirow{2}{*}{$\frac{10 \min }{\Delta \psi\left[^{\circ}\right]}$} & \multirow{2}{*}{$\frac{30 \min }{\Delta \psi\left[^{\circ}\right]}$} & \multirow{2}{*}{$\frac{70 \min }{\Delta \psi\left[^{\circ}\right]}$} \\
\hline & & & & & & & \\
\hline \multirow[t]{3}{*}{1} & 101 & 15 & 0 & 0 & 0 & 0 & 0 \\
\hline & 493 & 18 & 2 & 2 & 2 & 2 & 0 \\
\hline & 647 & 4 & 5 & 5 & 5 & 5 & 3 \\
\hline \multirow[t]{3}{*}{2} & 120 & 12 & 5 & 3 & 3 & 2 & 3 \\
\hline & 580 & 13 & 12 & 19 & 21 & 23 & 22 \\
\hline & 888 & 13 & 18 & 27 & 33 & 35 & 37 \\
\hline \multirow[t]{3}{*}{3} & 330 & 13 & 7 & 6 & 6 & 5 & 4 \\
\hline & 623 & 14 & 10 & 10 & 10 & 10 & 6 \\
\hline & 886 & 12 & 16 & 16 & 17 & 18 & 17 \\
\hline
\end{tabular}

Azimuthal rotation was counterclockwise for all cases. The uncertainty is $\delta \psi=0.5^{\circ}$

in Fig. 10. The curves show that the growth and final degree of rotation after $70 \mathrm{~min}$ is lower for thinner samples. For longer irradiation times a decrease in rotation was observed for some samples of polymer $\mathbf{1}$ and $\mathbf{3}$. We attribute this to change in optical birefringence after long irradiation durations, which leads to lower degrees of helical winding.

\section{The effect of PSSP on PSC}

In order to investigate the effect of inducing PSSP and PSC simultaneously, the samples were irradiated from the polymer side. Again, different layer thicknesses $d$ as well as exposure times of $10 \mathrm{~min}$ and $70 \mathrm{~min}$ were chosen. In this case, ellipticities of $\epsilon=15^{\circ}$ and $\epsilon=22.5^{\circ}$ were chosen for the incident EPL.

AFM images of those samples were taken to confirm PSSP upon irradiation with EPL; they are shown in Fig. 11. In case of $\epsilon=15^{\circ}$, the patterns seem more ordered and anisotropic. Otherwise, in case of $\epsilon=22.5^{\circ}$, the surface structures consist of randomly distributed bumps of the same shape. The larger disorder is attributed to the higher ellipticity, which means a closer polarization condition to
CPL. The shape of the bumps, however, differs slightly from the bumps achieved with LPL and CPL.

The polarimetry results for the azimuthal angle $\psi$ and azimuthal rotation $\Delta \psi$ for different $d, \epsilon$ and exposure times are listed in Table 4 and shown in Fig. 12. Different directions of azimuthal rotation result from an intentional change of the handedness of incident light and do not express a dependence on the sample parameters.

As can be seen in Table 4, the rotation angle differs not much from those obtained by irradiation from the glass side if the same ellipticity of $\epsilon=15^{\circ}$ is applied. This shows that anisotropic surface structures do not contribute significantly to the azimuthal rotation. Especially samples with polymer $\mathbf{2}$ are good examples due to the least pronounced surface structures with LPL and EPL. Moreover, a sample of this polymer showed one of the highest rotations with $23^{\circ}$ (irradiating azo-side for $10 \mathrm{~min}$, $d=580 \mathrm{~nm}, \epsilon=15^{\circ}$ ) and $37^{\circ}$ (irradiating glass-side for $70 \mathrm{~min}, d=580 \mathrm{~nm}, \epsilon=15^{\circ}$ ). Considering the specific sample with the thickness of $d=580 \mathrm{~nm}$ and irradiated with EPL ( $\epsilon=15^{\circ}$ for $\left.10 \mathrm{~min}\right)$, it is shown that the deviation of azimuthal rotation between the two irradiation

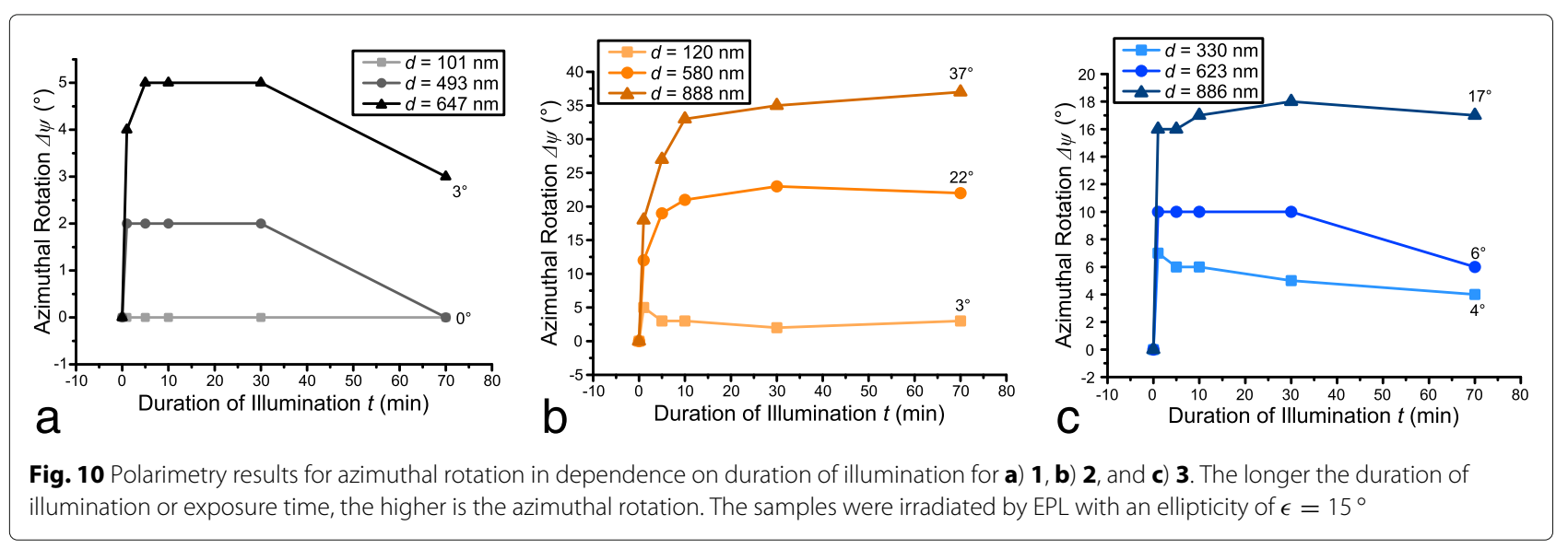




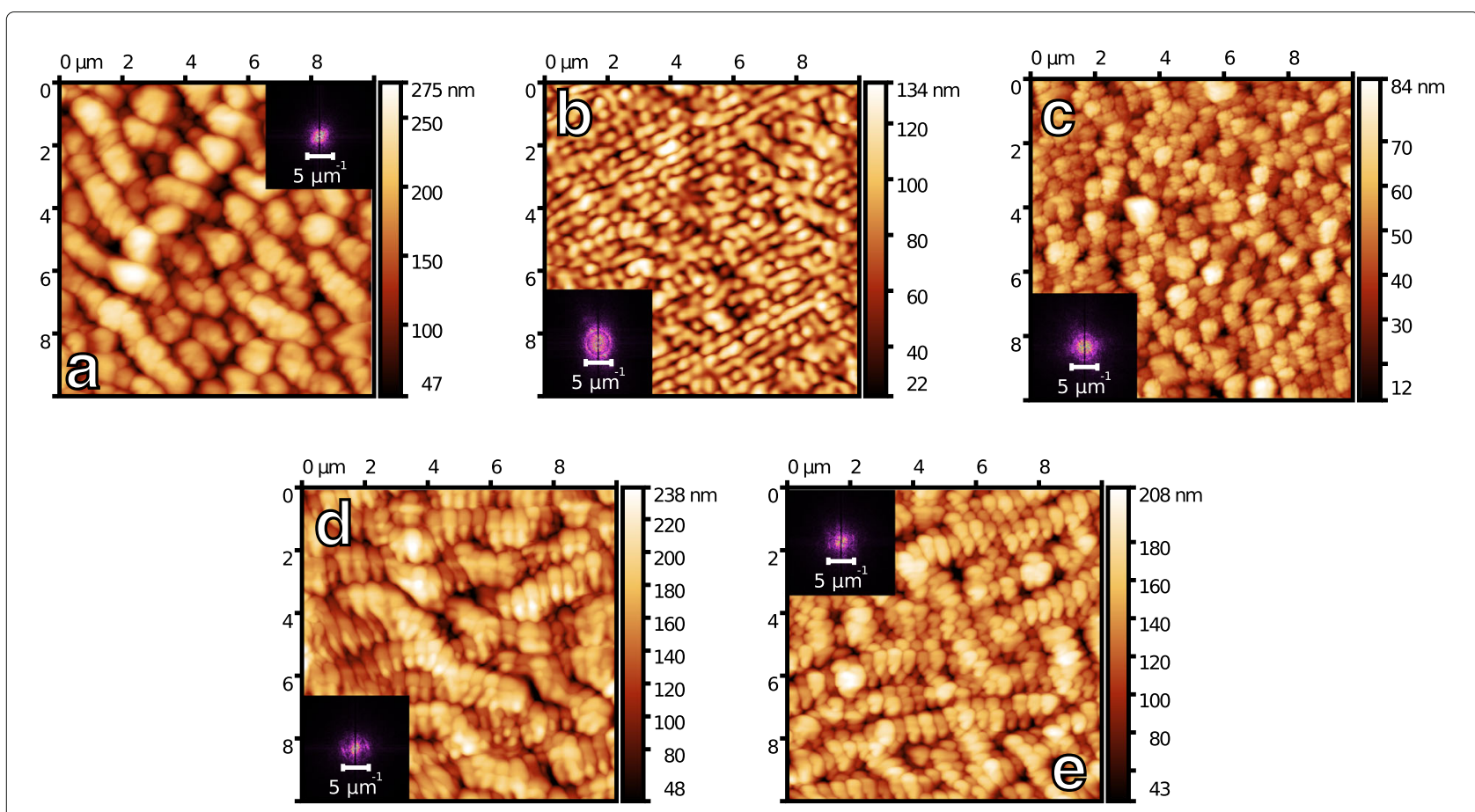

Fig. 11 AFM and FFT images of samples $\mathbf{a}) \mathbf{1}, \mathbf{b}), \mathbf{c}) \mathbf{2}$, and d), e) $\mathbf{3}$ after irradiating by EPL from the layer side. Various surface structures were obtained after irradiation by EPL with different $\epsilon$. Irradiation with $\epsilon=15^{\circ}$ (as seen in $\mathbf{b}$ ) and $\mathbf{d}$ )) leads to anisotropically oriented structures, and irradiation with $\epsilon=22.5^{\circ}$ in $\mathbf{a}$ ), $\mathbf{c}$ ), and e) leads to rather randomly distributed bump patterns. Here, the FFT images show higher order of surface structures. The AFM images show the condition after an exposure time of $70 \mathrm{~min}$

directions is very low with a value of $2^{\circ}$. Comparatively, this is a very low value and not significant in comparison to the high degrees of azimuthal rotation.

Going to a higher ellipticity of $\epsilon=22.5^{\circ}$, the angle of rotation decreases in case of polymer 2 , whereas higher rotations are observed with polymers 1 and $\mathbf{3}$. The highest azimuthal rotation of $\Delta \psi=59^{\circ}$ is obtained in case of 3 with the thickness of $d=886 \mathrm{~nm}$ and an exposure time of $10 \mathrm{~min}$ by using EPL with $\epsilon=22.5^{\circ}$. Also in this case, the azimuthal rotation decreases in most cases after longer exposure times until $70 \mathrm{~min}$, presumably due to the dynamic change of the birefringent helical structure. Another possible explanation for decreasing of rotation is that light-induced thermal effects caused by the high intensity of incident laser beam lead to relaxation of the inscribed helical arrangement. It is already well known that light-induced structuring of azo films is erasable by heating the sample above $T_{\mathrm{g}}[3-5,18,20]$.

Furthermore, it might be that photo-bleaching decreases the light-induced structuring of an azo layer.

Also, the transmission optics through helical structures may display maxima and minima with continuous change in birefringence as it was shown for liquid crystal displays by Gooch and Tarry in 1974 [23].

\section{Conclusion}

We have irradiated spin-coated azo polymers with linear, circular, and elliptically polarized light for different

Table 4 Results for polarimetry measurements in case of irradiating the azo layer side

\begin{tabular}{|c|c|c|c|c|c|c|c|}
\hline \multirow[t]{2}{*}{ Polymer } & \multirow[t]{2}{*}{$d[\mathrm{~nm}]$} & \multirow[t]{2}{*}{$\varepsilon\left[^{0}\right]$} & \multirow{2}{*}{$\begin{array}{l}0 \min \\
\psi\left[^{\circ}\right]\end{array}$} & \multicolumn{2}{|c|}{$10 \mathrm{~min}$} & \multicolumn{2}{|c|}{$70 \mathrm{~min}$} \\
\hline & & & & $\psi\left[{ }^{\circ}\right]$ & $\Delta \psi\left[^{\circ}\right]$ & $\psi\left[^{\circ}\right]$ & $\Delta \psi\left[^{\circ}\right]$ \\
\hline 1 & 493 & 22.5 & 4 & 16 & 12 & 15 & 11 \\
\hline \multirow[t]{2}{*}{2} & 580 & 15 & 12 & -11 & 23 & -7 & 19 \\
\hline & & 22.5 & 17 & 11 & 6 & 13 & 4 \\
\hline \multirow[t]{3}{*}{3} & 434 & 22.5 & 2 & 6 & 4 & 11 & 9 \\
\hline & 886 & 15 & 9 & -21 & 30 & -11 & 20 \\
\hline & & 22.5 & 16 & -43 & 59 & -24 & 40 \\
\hline
\end{tabular}

The uncertainty is $\delta \psi=0.5^{\circ}$ 

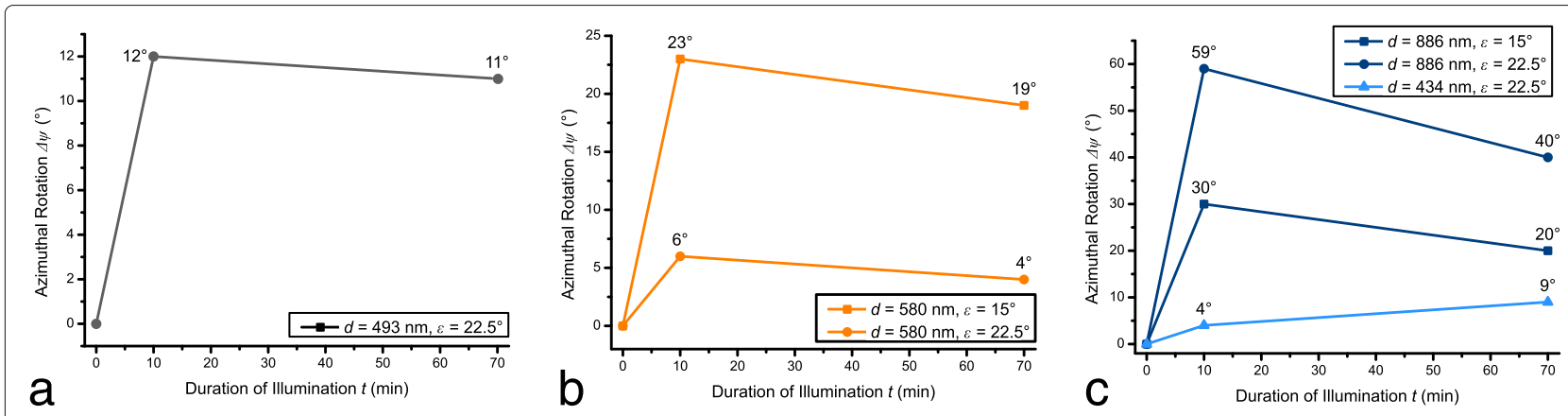

Fig. 12 Polarimetry results for irradiating the azo layer: a Polymer $\mathbf{1}, \mathbf{b}$ Polymer $\mathbf{2}$, and $\mathbf{c}$ Polymer $\mathbf{3}$. The longer the duration of illumination, the higher is the azimuthal rotation. However, the azimuthal rotation decreases in some cases after longer irradiation. The samples were irradiated by EPL with an ellipticity of either $\epsilon=15^{\circ}$ or $\epsilon=22.5^{\circ}$

durations of illumination and layer thicknesses. Photoinduced surface structuring (PSSP) as well as photoinduced supramolecular chirality (PSC) were produced successfully in all cases. The used high-performance three-core azo polymers (with high optical birefringence and polarizability) lead to very high values for PSC up to a rotation of $\Delta \psi=59^{\circ}$ for film thicknesses below $1 \mu \mathrm{m}$. We have shown that PSSP is not detrimental for the amount of optical rotation achieved in PSC. This would allow the production of optical devices with polarization optics and superhydrophobic surface patterns in one processing step, by using a single beam of polarized light.

\section{Experimental}

In order to treat and analyze the azo layers, the raw material was dissolved in different concentrations in tetrahydrofuran $(20,40$, or $80 \mathrm{mg} / \mathrm{mL})$ and stirred at ambient temperature $\left(20^{\circ} \mathrm{C}\right)$ for several days. The solutions were used for measuring absorption spectra with a Lambda 900 UV/VIS/NIR Spectrometer (PerkinElmer Instruments) as well as for spin-coating on $1 \mathrm{~cm} \times 2.5 \mathrm{~cm}$ glass substrates $(\sim 500-800 \mathrm{rpm})$, which have been cut out of an object slide. Film thicknesses have been measured with a spectroscopic ellipsometer (VASE ${ }^{\circ}$, J.A. Woollam Co., Inc.). The samples were dried for approx. 3 weeks in darkness at ambient temperature, so that it can be assured that azo molecules are in the thermodynamically stable trans-configuration.

The samples were treated with a continous wave DPSSlaser at $\lambda_{\text {exc. }}=473 \mathrm{~nm}$ and various polarization states (linear, circular and elliptic) for various irradiation durations $(1-70 \mathrm{~min})$; as seen in Fig. 4a. The average spot diameter of about $1.26 \mathrm{~mm}$ was determined with a Olympus E-3 camera after removing the objective in order to irradiate its CMOS-sensor with the laser spot. The fluence was $\approx 1200 \mathrm{~mW} / \mathrm{cm}^{2}$ and determined by measuring the absolute intensity with a power / energy meter (Vector $\mathrm{H} 410$, Scientech) and calculating with the determined spot diameter. The output power amounts to $36.7 \mathrm{~mW}$.
After light-treating, samples were characterized with a self-made polarimeter, which measures the angledependent intensity of transmitted light through a given sample. The polarimeter consists of a $\mathrm{HeNe}$-laser (Class III a, $4 \mathrm{~mW}$ ) at $\lambda=633 \mathrm{~nm}$, a linear polarizer (TECHSPEC ${ }^{\circ}$ glass polarization filters), a quarterwaveplate to switch the polarization state from linear to elliptic, the sample holder, the rotatable analyzer (same as polarizer) and a photodiode detector to measure the intensity of transmitted light; as seen in Fig. 2b. Data from the detector were collected by a self-written LabVIEW program, which also controlled the analyzer. Atomic force microscopy (AFM) images were measured with the JPK NanoWizard II from JPK Instruments. The raw JPK-files from the AFM measurements were corrected and analyzed with the software Gwyddion (v2.52). Corrections were made by using the polynomial method with a degree of 3. The color map for the height profile for each AFM image were done by using the "color range tool" and choosing the "automatic" option. Modulation depths were calculated from the histogram of the height profile with tails cut off, according to the color map.

\section{Abbreviations}

CPL: Circular polarized light; DPSS: Diode pumped solid state; EPL: Elliptically polarized light; LPL: Linear polarized light; PSC: Photoinduced supramolecular chirality; PSSP: Photoinduced spontaneous surface patterning; SRG: Surface relief grating

\section{Acknowledgements}

We gratefully thank Dr. S. Kostromine for synthesizing and providing the polymers.

\section{Funding}

Not applicable.

\section{Availability of data and materials}

The datasets used and/or analysed during the current study are available from the corresponding author on reasonable request.

\section{Authors' contributions}

SB performed the experiments. TF supervised the work. The text was written by both authors. Both authors read and approved the final manuscript. 


\section{Competing interests}

The authors declare that they have no competing interests.

\section{Publisher's Note}

Springer Nature remains neutral with regard to jurisdictional claims in published maps and institutional affiliations.

Received: 28 February 2019 Accepted: 3 May 2019

Published online: 18 June 2019

\section{References}

1. Fuhrmann, T., Salbeck, J.: Functional Molecular Glasses: Building Blocks for Future Optoelectronics. Adv. Photochem. 27, 83-166 (2002)

2. Fuhrmann, T., Salbeck, J.: Organic Materials for Photonic Devices. MRS Bull. 28(05), 354-359 (2003)

3. Rochon, P., Batalla, E., Natansohn, A.: Optically induced surface gratings on azoaromatic polymer films. Appl. Phys. Lett. 66(2), 136-138 (1995)

4. Kim, D. Y., Tripathy, S. K., Li, L., Kumar, J.: Laser-induced holographic surface relief gratings on nonlinear optical polymer films. Appl. Phys. Lett. 66(10), 1166-1168 (1995)

5. Yager, K. G., Barrett, C. J.: Light-induced nanostructure formation using azobenzene polymers. In: Nalwa, H. S. (ed.) Polymeric Nanostructures and their Applications, pp. 243-272. American Scientific Publishers, Los Angeles (2006)

6. Zilker, S. J., Bieringer, T., Haarer, D., Stein, R. S., Van Egmond, J. W., Kostromine, S. G.: Holographic data storage in amorphous polymers. Adv. Mater. 10(11), 855-859 (1998)

7. Perschke, A., Fuhrmann, T.: Molecular azo glasses as grating couplers and resonators for optical devices. Adv. Mater. 14(11), 841-843 (2002)

8. Fuhrmann, T., Tsutsui, T.: Synthesis and Properties of a Hole-Conducting, Photopatternable Molecular Glass. Chem. Mater. 11(8), 2226-2232 (1999)

9. Fuhrmann, T., Wendorff, J. H.: Optical Storage. Int. J. Polym. Mater. 45, 621-675 (2000)

10. Gruner, P., Arlt, M., Fuhrmann-Lieker, T.: Surface Wrinkling Induced by Photofluidization of Low Molecular Azo Glasses. ChemPhysChem. 14(2) 424-430 (2013)

11. Fuhrmann, T., Samse, K., Salbeck, J., Perschke, A., Franke, H.: Guided electromagnetic waves in organic light emitting diode structures. Org. Electron. 4(4), 219-226 (2003)

12. Reinke, N., Fuhrmann, T., Perschke, A., Franke, H.: Improved outcoupling of light in organic light emitting devices, utilizing a holographic DFB-structure. J. Lumin. 110(4), 413-417 (2004)

13. Goulet-Hanssens, A., Barrett, C. J.: Photo-control of biological systems with azobenzene polymers. J. Polym. Sci. Part A: Polym. Chem. 51(14), 3058-3070 (2013)

14. Barillé, R., Janik, R., Kucharski, S., Eyer, J., Letournel, F.: Photo-responsive polymer with erasable and reconfigurable micro- and nano-patterns: An in vitro study for neuron guidance. Colloids Surf. B: Biointerfaces. $\mathbf{8 8}$, 63-71 (2011)

15. Blair, H. S., Pague, H. I., Riordan, J. E.: Photoresponsive effects in azo polymers. Polymer. 21(10), 1195-1198 (1980)

16. Hubert, C., Malcor, E., Maurin, I., Nunzi, J. M., Raimond, P., Fiorini, C.: Microstructuring of polymers using a light-controlled molecular migration processes. Appl. Surf. Sci. 186, 29-33 (2002)

17. Hubert, C., Fiorini-Debuisschert, C., Maurin, I., Nunzi, J. M., Raimond, P.: Spontaneous patterning of hexagonal structures in an AZO-polymer using light-controlled mass transport. Adv. Mater. 14(10), 729 (2002)

18. Hubert, C., Fiorini-Debuisschert, C., Rocha, L., Raimond, P., Nunzi, J.-M.: Spontaneous photoinduced patterning of azo-dye polymer films: the facts. J. Opt. Soc. Am. B. 24(8), 1839 (2007)

19. Kim, M. J., Shin, B. G., Kim, J. J., Kim, D. Y.: Photoinduced Supramolecular Chirality in Amorphous Azobenzene Polymer Films. J. Am. Chem. Soc. 124(14), 3504-3505 (2002)

20. Kim, M. J., Yoo, S. J., Kim, D. Y.: A Supramolecular Chiroptical Switch Using an Amorphous Azobenzene Polymer. Adv. Funct. Mater. 16(16), 2089-2094 (2006)

21. Mazaheri, L., Lebel, O., Nunzi, J.-M.: Transfer of chirality from light to a Disperse Red 1 molecular glass surface. Opt. Lett. 42(23), 4845-4848 (2017)
22. Mazaheri, L., Rao Bobbara, S., Lebel, O., Nunzi, J.-M.: Photoinduction of spontaneous surface relief gratings on Azo DR1 glass. Opt. Lett. 41(13), 2958-2961 (2016)

23. Gooch, C. H., Tarry, H. A.: Optical characteristics of twisted nematic liquid-crystal films. Electron. Lett. 10(1), 2-4 (1974)

\section{Submit your manuscript to a SpringerOpen ${ }^{\circ}$ journal and benefit from:}

- Convenient online submission

- Rigorous peer review

- Open access: articles freely available online

- High visibility within the field

- Retaining the copyright to your article

Submit your next manuscript at $>$ springeropen.com 